\title{
Tactile localization performance in children with developmental coordination disorder (DCD) corresponds to their motor skill and not their cognitive ability
}

Article

Accepted Version

Creative Commons: Attribution-Noncommercial-No Derivative Works 4.0

Johnston, J. S., Begum Ali, J., Hill, E. L. and Bremner, A. J. (2017) Tactile localization performance in children with developmental coordination disorder (DCD) corresponds to their motor skill and not their cognitive ability. Human Movement Science, 53. pp. 72-83. ISSN 0167-9457 doi: https://doi.org/10.1016/j.humov.2016.12.008 Available at https://centaur.reading.ac.uk/68944/

It is advisable to refer to the publisher's version if you intend to cite from the work. See Guidance on citing.

To link to this article DOI: http://dx.doi.org/10.1016/j.humov.2016.12.008

Publisher: Elsevier

All outputs in CentAUR are protected by Intellectual Property Rights law, including copyright law. Copyright and IPR is retained by the creators or other copyright holders. Terms and conditions for use of this material are defined in the End User Agreement. 


\section{www.reading.ac.uk/centaur}

\section{CentAUR}

Central Archive at the University of Reading

Reading's research outputs online 
RUNNING HEAD: Tactile localization in DCD

Tactile localization performance in children with developmental coordination disorder (DCD) corresponds to their motor skill and not their cognitive ability

Joanne S. Johnston ${ }^{1,2}$, Jannath Begum Ali ${ }^{1,3}$, Elisabeth L. Hill ${ }^{1}, \&$ Andrew J. Bremner ${ }^{1}$

${ }^{1}$ Sensorimotor Development Research Unit, Department of Psychology, Goldsmiths, University of London, London SE14 6NW, UK.

${ }^{2}$ Department of Psychology, School of Psychology and Clinical Language Sciences, University of Reading, RG6 7BE, UK

${ }^{3}$ Centre for Brain and Cognitive Development, Department of Psychological Sciences, Birkbeck University of London, Malet Street, London, WC1E 7HX.

SUBMITTED TO: HUMAN MOVEMENT SCIENCE (FEBRUARY, 2016)

ABSTRACT WORD COUNT: 222

WORD COUNT: 6,791 WORDS (Excluding Reference List)

REFERENCES: 48

CORRESPONDING AUTHOR: Dr Joanne S. Johnston (née Camp), Department of Psychology, School of Psychology and Clinical Language Sciences, University of Reading, RG6 7BE, UK.; Tel.: +44 (0) 118378 8523; Fax.: +44 (0) 118378 6715; email:

j.camp@gold.ac.uk / jo.johnston@ reading.ac.uk 


\begin{abstract}
When localizing touches to the hands, typically developing children and adults show a "crossed hands effect" whereby identifying which hand received a tactile stimulus is less accurate when the hands are crossed than uncrossed. This demonstrates the use of an external frame of reference for locating touches to one's own body. Given that studies indicate that developmental vision plays a role in the emergence of external representations of touch, and reliance on vision for representing the body during action is atypical in developmental coordination disorder (DCD), we investigated external spatial representations of touch in children with DCD using the "crossed hands effect". Nineteen children with DCD aged 7-11 years completed a tactile localization task in which posture (uncrossed, crossed) and view (hands seen, unseen) were varied systematically. Their performance was compared to that of 35 typically developing controls (19 of a similar age and cognitive ability, and 16 of a younger age but similar fine motor ability). Like controls, the DCD group exhibited a crossed hands effect, whilst their overall tactile localization performance was weaker than their peers of similar age and cognitive ability, but in line with younger controls of similar motor ability. For children with movement difficulties, these findings indicate tactile localization impairments in relation to age expectations, but apparently typical use of an external reference frame for localizing touch.
\end{abstract}


KEYWORDS: DEVELOPMENTAL COORDINATION DISORDER; TOUCH; TACTILE LOCALIZATION; BODY REPRESENTATIONS; MULTISENSORY DEVELOPMENT; SENSORIMOTOR DEVELOPMENT.

Highlights

- Children with DCD exhibit a crossed hands effect like typically developing controls

- Tactile localization ability is immature in children with DCD

- Children with DCD use an external frame of reference for localizing touch 


\section{Introduction}

Integrating information from multiple senses facilitates the construction of a representation of the body with respect to the external world which is necessary for effective interaction with one's immediate environment, such as through motor control (see Bremner, Holmes, \& Spence, 2008). For example, in order to make a successful reach for a rattle, an infant needs to be able to understand where that rattle is in relation to her own arm, perhaps using information from vision, audition, and/or proprioception. Early abilities, and indeed impairments in these multisensory processes, are likely to have downstream effects in later development (Hill, Crane, \& Bremner, 2012; Karmiloff-Smith, 1998), making them important when thinking about the development of motor (and other) domains: for example, if the infant reaching for the rattle (mentioned above) successfully executes her reach, she will be presented with an opportunity for learning about the object.

During development, there are significant challenges to such multisensory representations of the body, as they must be maintained and continually updated, not only in the face of changes in body posture (e.g., across different limb positions), but also to take account of changes in the size and proportion of the body and limbs as they change throughout childhood (see Bremner, Holmes, \& Spence, 2012). However, by the time adulthood is reached, it is thought that we are able to make use of statistical information from the senses in an optimally weighted pattern, such that individual senses are relied upon for spatial representations in accordance with how reliable their sensory information is for a given situation or task (e.g., Ernst \& Banks, 2002).

Recent findings indicate that, early in childhood, children use sensory cues in a more separate fashion for sensorimotor performance than do adults, for instance by relying on one source of sensory input, rather than a combination of cues (Bremner \& De Fockert, 2016; Gori, Del Viva, Sandini, \& Burr, 2008; Nardini, Begus, \& Mareschal, 2013; Nardini, Jones, 
Bedford, \& Braddick, 2008). One phenomenon which is often reported in early childhood is referred to as "visual dominance", in which visual cues are more strongly weighted relative to those arising from proprioception. Greater visual weighting is often seen in adults, where visual cues in many contexts are more reliable for a given task, but such visual reliance can be greater in early childhood (Cowie, Makin, \& Bremner, 2013; Cowie, Sterling, \& Bremner, 2016; Sparto et al., 2006; Warren \& Pick, 1971) and is not necessarily explained by the poorer reliability of the other senses (Gori et al., 2008).

Another group of individuals who are thought to overly depend on visual inputs are children with developmental coordination disorder (DCD). DCD is a neurodevelopmental disorder characterized by an impairment in movement in relation to the level that would generally be expected on the basis of a child's chronological age and intellectual ability, and which negatively affect activities of daily living or academic achievement, in the absence of a neurological or intellectual impairment (APA, 2013). Children with DCD can be hyper- or hypo-sensitive to individual sensory inputs (such as sound or touch) although this is by no means unique to the disorder, and is found across atypically developing populations (Cascio, 2010; Hill et al., 2012). In unisensory deficit accounts of the disorder, difficulties processing information from individual sensory channels such as vision (e.g., Lord \& Hulme, 1987) or proprioception (e.g., Laszlo, Bairstow, Bartrip, \& Rolfe, 1988) have been argued to be instrumental in the difficulties with motor control which children with DCD experience. However, given that the senses do not operate in isolation either in adulthood (e.g., Spence \& Driver, 2004), or in early life (Bremner et al., 2012), it is almost certain that they impact upon and are impacted by each other during development. As such, an early unisensory impairment could lead to downstream multisensory impairment. Furthermore, an early multisensory impairment could lead to downstream unisensory deficits (Hill et al., 2012). It is clear that the difficulties faced by children with DCD should be considered in a multisensory context. 
Considerable progress has been made in the field in terms of examining multisensory processes which occur during the performance of sensorimotor tasks in DCD. Evidence for problems with the tuning of multisensory inputs in DCD arises from investigations utilising multisensory experimental tasks (e.g., von Hofsten and Rösblad, 1988; see below) as well as from investigations concerning the reliance on various sensory inputs for more generalized motor performance: for example, children with DCD rely on visual information disproportionately for sensorimotor control of gross motor tasks like walking, balance and reaching (Deconinck et al., 2006; Wann, Mon-Williams, \& Rushton, 1998). Moreover, the swinging room task which measures the interaction of visual and proprioceptive cues aiding balancing indicates that illusory visual cues to self motion are not appropriately moderated by veridical proprioceptive cues, leading to a greater negative impact of the swinging room on the balance of a subset of children with DCD who had postural control difficulties compared to typically developing (TD) controls (Wann et al., 1998).

The interaction of visual and proprioceptive cues in reaching tasks has been examined in a task developed by Von Hofsten and Rösblad (1988). This task presents children with a spot on a tabletop that could be either seen (visually - by looking), felt (proprioceptively - by touching), or both (by looking and touching), and asked them to stick a pin underneath the table in the same position as the spot. Children with DCD have been found, in several studies (e.g., Mon-Williams, Wann, \& Pascal, 1999; Schoemaker et al., 2001), to perform worse than their peers in general at the task. However, poorer performance on the visual condition by the DCD group was found by Mon-Williams et al. (1999), suggesting that there is a particular impairment in DCD concerning the ability to link visual information (on top of the table in this task) to proprioceptively controlled reaching (under the table in this task). Thus, across a range of sensorimotor tasks, children with DCD seem to show atypicalities in the ways in 
which visual and proprioceptive cues interact to form representations of the layout of their limbs and bodies.

Rather than focusing on the visual-proprioceptive processes which are so central to most sensorimotor tasks in the literature, in this study we examined body representations in DCD in a more perceptual context. We assessed tactile localization - the ability to locate specific touches on the body. A number of studies now demonstrate that tactile localization is a fundamentally multisensory process. Although locating a touch on the body surface could theoretically be achieved simply through registering that touch with respect to a somatosensory body map, in many situations we also locate touches to places in the external spatial environment (Medina \& Coslett, 2010). Many studies now demonstrate that visual, proprioceptive and even auditory cues contribute to localizing touches in external space in adults (e.g., Pavani, Spence, \& Driver, 2000; Rigato et al., 2013; Tajadura-Jiménez, Väljamäe, Toshima, Kimura, Tsakiris \& Kitagawa, 2012), and even in infants (Begum Ali, Spence, \& Bremner, 2015; Rigato, Begum Ali, Van Velzen \& Bremner, 2014).

With the use of tactile temporal order judgement (TOJ) tasks, it has been possible for researchers to measure participants' use of an external reference frame for tactile localization (Heed \& Azañón, 2014). In these tasks, participants are typically asked to identify which of two hands (or feet) were touched first (e.g., by pressing a button with the hand which was touched first). By varying the position of these limbs in external space, somatotopic and external frames of reference can be put into conflict (the most typical manipulation is to cross the hands), and so errors are predicted under such circumstances if the participant is using an external frame of reference.

The "crossed hands effect", in which poorer tactile localisation accuracy is seen when the hands are crossed, is seen in human adults, children and infants in a range of contexts (e.g., Azañón, Stenner, Cardini, \& Haggard, 2015; Pagel et a., 2009; Begum Ali et al., 2014; 
Bremner et al., 2008; Schicke \& Röder, 2006; Shore, Spry, \& Spence, 2002; Yamamoto \& Kitazawa, 2001) and is used as an index of the participant's reliance on an external frame of reference for coding touch. The crossed hands effect is not only seen in blindfold sighted adults, but also after many years of acquired blindness (e.g., Bremner \& Van Velzen, 2015; Röder, Rosler, \& Spence, 2004) although not in congenitally blind adults (Röder, Rosler, \& Spence, 2004). This indicates that visual experience plays an important role in the development of external spatial representations of touch.

More recently, researchers have begun investigating the external spatial coding of touch in typical development. Using a tactile TOJ task, Pagel, Heed and Röder, (2009) showed that children from 5.5 years exhibited a crossed hands effect. However, evidence is now mounting that external spatial representations of touch have their origins in human infancy with crossed hands and feet effects emerging in tactile orienting responses by 6 months of age (Begum Ali, Cowie, \& Bremner, 2014; Begum Ali, Spence, \& Bremner, 2015; Bremner, Mareschal, Lloyd-Fox, \& Spence, 2008). Additionally, Begum Ali et al. (2014) report that sight of the hands and arms made tactile localization more difficult for 4-year-olds when their hands were in a canonical posture (uncrossed). This indicates early difficulties in typical development with integrating visual signals into the body schema (Begum Ali et al., 2014). It appears likely that early multisensory experience of visual tactile events drives the development of an ability to perceive touches in external space (Bremner \& Spence, in press).

In light of previous evidence of atypical multisensory body representations in DCD with respect to the way that vision and proprioception underlie impaired motor control in DCD (see Hill et al., 2012), the purpose of the present study was to extend the investigation of body representations in DCD to the broader and more multisensory perceptual paradigm of spatial tactile localisation. In order to pinpoint the development of tactile localisation abilities in DCD, we compared our DCD group to two control groups: one of an equivalent age and 
cognitive ability and one younger TD group of children with equivalent fine motor skill to the DCD group. There is evidence that the use of an external frame of reference for localizing touch is dependent upon visual experience (Röder et al., 2004) in early life (Begum Ali et al., 2015) and that young children have difficulty integrating visual signals into the body schema for tactile localisation (Begum Ali et al., 2014). In considering the importance of bodily representations for action (and the action impairments inherent in DCD), we viewed it important to characterise the extent to which DCD children show typical abilities in localising touches on the body and in external space. In order to understand how other domains may be contributing to tactile localisation in DCD, we also investigated whether there were tactile localisation associations with fine motor skill or attentional capability.

\section{Method}

We examined localization of touches applied to the hands in children with DCD, comparing their performance across crossed-hands and uncrossed-hands with reference to the performance of typically developing peers. In order to make the task accessible for a wide range of age groups, rather than the tactile temporal order judgment task we used a simplified tactile localization task developed by Begum Ali, Cowie, \& Bremner (2014). This task presents one tactile stimulus instead of two, requiring children to report which hand was touched per se rather than which hand was touched first. We also varied systematically whether or not the participants could see their own arms by either covering them up or leaving them visible.

Children with DCD aged 7-11 years, and two control groups of either equivalent chronological age and cognitive ability (CAIQ group), or younger age and equivalent fine motor skills (motor matched, MM, group), completed a task in which they were asked to identify which of their hands a vibrotactile stimulus was presented to. Each of their hands was stimulated with a vibrotactile tactor which was in turn placed just below a toy furry 
animal (either a penguin or a hedgehog). The participants were asked to identify which of the two animals "tickled" their hands. They received 20 trials in the uncrossed-hands posture and 20 trials in the crossed hands posture. This was repeated in visible and covered blocks of trials, in a counterbalanced order. Participants also completed tests of cognitive and motor ability and parents completed questionnaires concerning background, attentional and motor characteristics, in order to inform group matching and individual differences with regards to task performance.

\subsection{Participants}

\subsubsection{Recruitment}

The study was conducted as part of a larger investigation into multisensory development. Parents provided written informed consent for their child's participation. The research was approved by the research ethics committee at Goldsmiths, University of London. Children with an existing diagnosis of developmental coordination disorder (DCD) or dyspraxia were recruited through families who were known to the Goldsmiths Action Lab because they were taking part in other research projects. The typically developing (TD) children were either recruited in the same way as the DCD group or through links with local schools.

\subsubsection{DCD group}

Criteria for DCD according to the Diagnostic and Statistical Manual, $5^{\text {th }}$ edition (DSM-V, APA, 2013) require that a) motor performance is poor in relation to chronological age and opportunity for skill acquisition; b) it impacts on activities of daily living or academic achievement; c) motor difficulties have an early onset; d) that motor difficulties are not better explained by visual impairment, intellectual or neurological conditions. We addressed each of the criteria in the following ways. For criterion (a), we gathered information from parents (with the help of the class teacher in one case) about their child's 
motor abilities using the Movement Assessment Battery for Children $-2^{\text {nd }}$ edition Checklist (MABC-2 Checklist; Sugden \& Henderson, 2007). A score on this measure is based on responses to items describing 30 everyday motor tasks (comprising both fine and gross motor skills) for which parents rate their child's level of proficiency (Very Well, Just OK, Almost or Not Close). The questionnaires were scored as per the instructions in the manual, with the exception that where a parent circled two adjacent options (e.g., 1 and 2) a score of the midpoint between these was awarded (e.g., 1.5) rather than treating the item as a missing response. The criterion for inclusion in the DCD group was a Checklist score at or above the $95^{\text {th }}$ percentile. As an additional confirmation of motor difficulties, all but two of our participants with DCD had full MABC-2 test scores valid from a previous visit to the lab (including one participant whose parent did not complete any questionnaires). Participants with scores above the $16^{\text {th }}$ percentile $(n=1)$ were excluded, and thus, criterion (a) was established for all participants through the MABC-2 test, Checklist, or both. Parents confirmed through completion of a questionnaire that motor difficulties were apparent early in development and impacted upon activities of daily living, thus confirming criteria (b) and (c). With regard to criterion (d), none of the DCD participants were reported to have diagnoses of visual, or neurological impairments (such as cerebral palsy). To establish an absence of intellectual disability, an inclusion criterion for any participant in the study (DCD or TD) was a minimum standardized score of 70 on measures of both receptive vocabulary (British Picture Vocabulary Scale, 3rd edition; BPVS-III, Dunn et al., 2009) and nonverbal ability (Raven's Coloured Progressive Matrices; RCPM, Raven, 2004). Two children with DCD scored below this cut-off and were excluded from the sample.

In order to gain an understanding of the potential impact of children's attentional characteristics on their performance, parents were also asked to complete the Conners 3 ADHD rating scale (Conners, 2008; 2009; described further in section 2.2.4) 
Of the final DCD sample of 19 , the following numbers of children in the DCD group had co-occurring difficulties: attention deficit disorder (1), sensory processing difficulties (8), of whom 2 had Irlen syndrome, anxiety (2), present or previous problems with speech/speech and language, including reading difficulties and verbal dyspraxia (5). Children with an additional diagnosis of autism were not recruited.

\subsubsection{Control groups}

TD children were included if their scores fell below the 95th percentile on the MABC-Checklist, indicating performance in the top 94\% of their age cohort. Parents of five and seven participants in the CAIQ and MM groups respectively did not return the Movement-ABC Checklist. However, for all typically developing participants, parents confirmed the absence of any medical conditions (e.g., physical, cognitive or neurological condition; visual, speech, hearing or other sensory impairments; anxiety problems, etc., with the exception of needing to wear glasses to correct vision) or developmental disorders (e.g. autism spectrum disorder, ADHD, dyslexia, developmental co-ordination disorder/dyspraxia, specific language impairment), even when they did not return the questionnaires. Therefore, they were still included in the dataset, as we can be confident that they were indeed typically developing. In addition, the same inclusion criteria of a minimum standardized score of 70 on the BPVS and RCPM was applied to the TD children, as for the DCD group.

We compared the performance of the participants with DCD to that of two control groups. One group (the CAIQ group) were individually matched to the participants with DCD on the basis of their raw score on the RCPM (out of a maximum of 36). All children were matched to within 3 points, with the majority (73.7\%) matched to 0 or 1 point. As a group, both their verbal scores on the BPVS and their chronological age was equivalent to that of the DCD group (see Table 1). 
A second group of younger TD children (aged 5-6 years) were screened for fine motor skills to act as a motor match (MM) group, in a similar way to the published literature (e.g., Hulme, Smart, Moran, \& McKinlay, 1984; Sinani, Sugden, \& Hill, 2011). The MM group represented a control group whose fine motor ability was equivalent to that of the DCD group, but was typical for the child's age. To index fine motor functioning, we measured the speed with which participants placed 12 plastic 'mushroom'-shaped pegs into a pegboard, following a practice trial (for each hand) placing 6 pegs, as an average of the time taken for one trial using each of their preferred and non-preferred hands. By additionally including a motor-matched comparison group, we will gain a more nuanced picture of the abilities of the DCD group on an experimental task: i.e., how the DCD group's performance compares not only to the level that would be expected for their age and cognitive ability, but also to the level expected for typically developing children of equivalent motor skill. This approach also supports interpretation of the findings with regard to whether DCD performance can be considered typical but immature, or whether qualitatively different performance is exhibited. We chose to match this younger control group on fine motor skills as it was thought to be most relevant to the experimental task (e.g., compared to gross motor skills), in which children remain seated and attend to their hands.

\subsubsection{The Conners 3 attention questionnaire}

In order to gain an understanding of the potential impact of children's attentional characteristics on their performance, parents were also asked to complete the Conners 3 ADHD rating scale (Conners, 2008; 2009). Parents of children aged 6 years and over completed the ADHD Index version and parents of children aged 5 years completed the Early Childhood version, as appropriate. Both questionnaires are short versions of the full length questionnaire assessing attentional skills, and consist of 10 items for which the parent chooses how true each description of behaviour has been for their child over the past month, 
from 0 (Not true at all/Never, Seldom) to 3 (Very much true/Very often, very frequently). Three participants (in the MM group) were 5 years old and their parents completed the EC version, whilst all of the remaining participants had the 3AI version completed for them. For the DCD group ( $\mathrm{n}=18$ as one parent did not complete any questionnaires), the majority of the standardized scores were at or above $90(n=15)$, which is the maximum provided in the manual. This indicates a high level of symptoms related to ADHD. For the CAIQ matched group ( $\mathrm{n}=14$ for questionnaires) and MM group ( $\mathrm{n}=9$ for questionnaires), standardized scores ranged from 44 to 82 and from 40 to 69 respectively. T scores of $\geq 65$ are indicative of an elevated score (i.e., poor attention). The percentages of the DCD, CAIQ and Motor matched groups with returned questionnaires with a $\mathrm{T}$ score of 65 or above were: DCD: 17/18 (94.4\%), CAIQ: 3/14 (21.4\%); Motor matched: 1/9 (11.11\%).

$\underline{\text { Demographic characteristics of the final sample }}$

The final sample comprised 54 participants: 19 with DCD, 19 in the CAIQ group, and 16 in the MM group. One-way ANOVAs were conducted on CA, RCPM raw score, BPVS raw score and peg placing time, for which all were significant at $p<.001$. Tukey post hoc tests were conducted to compare groups for matching purposes, outcomes of which are contained in Table 1. The group differences were all as required by the selection criteria: i.e., the MM group were significantly younger, with lower cognitive ability raw scores than the DCD and CAIQ groups, who did not differ from one another on these measures; for peg placing times, the CAIQ group were faster than the DCD and MM groups, who did not differ from one another. Summary demographic characteristics are available in Table 1.

--Insert Table 1 about here-

\subsection{Materials and Apparatus}

An EPRIME script run by a laptop computer controlled the signal outputs $(220 \mathrm{~Hz}$ sine wave) to two voice coil vibrotactile transducers ("tactors"; $15 \times 15 \times 15 \mathrm{~mm}$ ). These 
tactors were attached with Velcro to the right and left hand side of a wooden platform measuring approximately $510 \mathrm{~mm}$ by $110 \mathrm{~mm}$ and. The tactors were placed $325 \mathrm{~mm}$ apart and $35 \mathrm{~mm}$ from the back of the platform. The participants were asked to rest their fingers comfortably on these two tactors. The wooden platform fitted underneath a custom-made Perspex stand, covered with black faux fur (see Fig. 1) measuring approximately $530 \mathrm{~mm}$ across the top edge, and $90 \mathrm{~mm}$ in height. A small toy penguin and hedgehog, each measuring approximately $110 \mathrm{~mm}$ in height, were attached to the top of the stand, directly above the position of the tactors on the wooden platform underneath. The penguin was always positioned on the left hand side, from the participant's perspective, with the hedgehog positioned on the right hand side. A length of Velcro (visible in Fig. 1) was attached to the front of the stand. In the Unseen conditions, a detachable cover made of the same black faux fur was placed over the participant's hands and arms, so that the appearance was of a continuous expanse of black fur between the participant and toy animals. An MP3 player attached to a small speaker played continuous pink noise at a volume of approximately 55 $\mathrm{dBA}$, in order to mask any audible sounds (approx. $49.7 \mathrm{dBA}$ ) made by the tactors. The apparatus are displayed in Figure 1.

--Insert Figure 1 about here--

\subsection{Design and Procedure}

Participants reported which toy animal 'tickled' their finger in a 3 x 2 x 2 mixed design of Group (DCD; CAIQ; MM), systematically varied limb position (Posture: Crossed vs. Uncrossed) and visibility (View: Visible vs. Covered), producing four unique conditions (Visible / Uncrossed; Visible / Crossed; Covered / Uncrossed; Covered / Crossed). There were 20 trials in each of the four blocks, corresponding to the four conditions, and therefore 80 trials in total. In each trial, one of the two tactors was triggered by the computer in a random order, so that each possible response ("penguin" or "hedgehog") was correct on half 
of the trials in each block. Participants completed all four conditions in one of four counterbalanced orders: i) Uncrossed Visible, Crossed Visible, Uncrossed Covered, Crossed Covered; ii) Crossed Visible, Uncrossed Visible, Crossed Covered, Uncrossed Covered; iii) Uncrossed Covered, Crossed Covered, Uncrossed Visible, Crossed Visible; iv) Crossed Covered, Uncrossed Covered, Crossed Visible, Uncrossed Visible.

Testing took place either in a quiet room at the participant's school, or in the university. The task was introduced to each participant as a game. Participants were first asked to say the name of the two toy animals to ensure that they were familiar with the object labels, and on the rare occasion that 'penguin' or 'hedgehog' was not immediately provided, the experimenter named the animal and asked the participant to repeat it until satisfied that they were readily able to produce it. The participant was shown the tactors which were underneath the animals (these were called "buzzers" to the participant) and it was explained to the child that one of the animals was going to tickle their finger each time, and that their task was to say which one tickled them. The experimenter helped the participant to put their two index fingers onto the buzzers in a Visible, Uncrossed posture.

The task began with a short practice phase of six trials (three of each hand) in the uncrossed, seen condition, which served to familiarise participants with the task and to prevent progression onto the experimental trials before a certain level of accuracy had been reached. Each trial began with the participant resting their index fingers on the tactors. The experimenter pressed a key on the laptop keyboard to trigger a gentle vibrotactile stimulus to one of the two tactors (i.e., situated underneath the penguin or the hedgehog). Each vibrotactile stimulus was presented for $200 \mathrm{~ms}$. The participant reported which tactor they felt vibrating, by verbalising either 'penguin' or 'hedgehog'. The experimenter coded the participant's responses on the computer. Participants were required to answer five or six trials correctly before continuing to the experimental phase. Although the practice trials were 
repeated if necessary, the vast majority of participants achieved five or six correct trials on their first attempt.

The experimental trials proceeded in an identical fashion to the practice trials, with the exception that the experimenter was now blind to the correct response. The participant's first answer was taken, unless they changed their answer before the experimenter had inputted it to the computer. If the participant answered that they did not know, after being encouraged to guess, the trial was disregarded in the analysis.

At the end of every block, the participant's contribution was praised and the experimenter adjusted the set-up of the apparatus according to the next block that was to be run: the black faux fur cover was added or removed to reveal or cover the participant's hands, and/or the participant's arms were adjusted (by the experimenter) to a crossed (with right arm over left arm) or uncrossed position. Participants were asked to close their eyes while the adjustments were performed. The experimenter checked that the participant was comfortable and ready to continue before the next block was administered. The whole task took approximately 10 minutes to run for each participant. Participants received a sticker as a reward for completing the task (where this was permitted by their school) and a certificate for taking part in the study overall.

\subsection{Data analysis}

The participants' performance in each condition was operationalised as the percentage of trials answered correctly. Percentage accuracy was averaged across the four conditions for each participant, to produce a measure of overall accuracy. The descriptive statistics for performance on each condition are displayed in Table 2. Trials in which the participant did not choose either the penguin or the hedgehog were excluded, as was one trial in which one participant gave an ambiguous answer. In total, 11 trials $(0.25 \%$ of all trials $)$ were excluded in this way. 
Tactile localisation data did not meet the assumption of normality for parametric testing (this is likely to be because accuracy was generally high). Transformations were unsuccessful in producing normality in the dataset, and so nonparametric analyses were employed. Accuracy was compared across levels of Group (DCD / CAIQ / MM), View (Unseen / Seen) and Posture (Uncrossed / Crossed) in order to investigate the presence and nature of the crossed hands effect in our DCD sample. The significance levels reported below are (where relevant) exact and two-tailed. Effect sizes (absolute values) for each test were calculated using $r(\mathrm{Z} / \sqrt{ } \mathrm{N})$. One score for a participant with DCD was an extreme outlier in terms of overall accuracy and exhibited a particularly low score on the Covered, Crossed condition. The analysis was re-run excluding this participant and the pattern of results was unchanged, so the analyses reported include this participant.

Correlations were conducted between tactile localisation accuracy and individual difference measures of attention and manual dexterity (Conners ADHD Index questionnaire scores and peg placing time) in order to examine potential explanations for performance.

\section{--Insert Table 2 about here--}

\section{Results}

\subsection{Tactile localisation across groups}

Descriptive measures of tactile localisation are displayed in Table 2. In light of the proximity of the mean scores to $100 \%$ (ceiling), we compared the accuracy of experimental task performance to $100 \%$ using a Wilcoxon signed ranks test, for each condition, for each group separately. The following conditions were not significantly different from 100\% (i.e., were at ceiling): for the MM group, Unseen Uncrossed $(p=.066)$; for the CAIQ group, Unseen Uncrossed $(p=.157)$, Seen Crossed $(p=.102)$, Seen Uncrossed $(p>.999)$. All other mean scores (including each condition for the DCD group) were significant, i.e., below 
ceiling $(p<.05$ for all). There was no significant main effect of counterbalancing order $\left(\right.$ Kruskal-Wallis: $\left.\operatorname{chi}^{2}(3)=.172, p=.982, r=.29\right)$.

Percentage accuracy differed significantly across DCD, CAIQ and MM groups, as indicated by a Kruskal-Wallis test $\left(\mathrm{chi}^{2}(2)=17.47, p<.001\right)$. Follow up Mann-Whitney U tests ( $\mathrm{p}$ values were multiplied by 3 to address type 1 error) indicated significantly lower accuracy in the DCD group compared to the CAIQ group $(U=77, p=.006, r=.51)$ and significantly lower accuracy in the MM group compared to the CAIQ group $(U=36.5, p=$ $.003, r=.67)$. The DCD and MM groups' accuracy did not differ significantly $(U=120.5, p$ $=.903, r=.18)$.

In order to examine the effects of View (Unseen, Seen) and Posture (Uncrossed, Crossed) on performance, means were calculated across the relevant conditions (i.e., regardless of group membership). A Wilcoxon signed ranks test indicated that Seen conditions $(M d n=100)$ were completed with equivalent accuracy to Unseen conditions $(M d n$ $=97.5), z=-.848, p=.396, r=0.12$. However, Crossed conditions $(M d n=97.5)$ were completed with lower accuracy than Uncrossed conditions $(M d n=100), z=-2.969, p=.003$, $r=.4$

The effect of Posture (i.e., the difference in accuracy between the Crossed and Uncrossed conditions) was compared for the Visible and Covered conditions, using a Wilcoxon test, which was non-significant $(\mathrm{Z}=-.559, p=.576, \mathrm{r}=.08)$, suggesting a lack of View by Posture interaction.

The interaction of View (Seen / Unseen) x Group (DCD / CAIQ / MM) was examined by calculating the difference between the average of the Seen and Unseen conditions for each participant, and comparing this across groups. A Kruskal-Wallis test indicated that there was no significant difference in this score across groups $\left(\operatorname{chi}^{2}(2)=.166, p=.920, \mathrm{r}=.19\right)$, indicating no interaction of View $\mathrm{x}$ Group. We also examined the interaction of Posture $\mathrm{x}$ 
Group, in the same way, calculating the difference between the average of the Uncrossed and Crossed conditions for each participant. Again there was no significant difference in this score across groups $\left(\mathrm{chi}^{2}(2)=3.301, p=.192, \mathrm{r}=.16\right)$ indicating no interaction of Posture $\mathrm{x}$ Group. The View x Posture x Group interaction was examined using the following formula for each participant, and compared across groups: ([Seen/Uncrossed $]-[$ Seen/Crossed $])-$ ([Unseen/Uncrossed] - [Unseen/Crossed]). The difference in this score across groups was not significant $\left(\mathrm{chi}^{2}(2)=.381, p=.827, \mathrm{r}=.13\right)$, indicating that there was no 3 way interaction. Figure 2 displays the accuracy by Posture and Group, collapsed across View.

--Insert Figure 2 about here--

\subsection{Individual differences}

The effect of gender on tactile localization accuracy was non-significant ( $U=333.5, p$ $=.739, r=.05)$. In order to examine the impact of attention on task performance, and the relationship between individual differences in motor skills and task performance, the following Spearman's correlations were conducted: a) between overall tactile localization accuracy and raw scores on the Conners ADHD Index (measuring parent-reported attentional skills); b) between overall tactile localization accuracy and average peg placing time (indexing fine motor skills). Each of these correlations was conducted for each experimental group separately (DCD; CAIQ; MM); however, a peg placing time correlation was not conducted for the MM group because they were recruited on the basis of this test. Raw scores were used for the Conners questionnaire correlations in place of standardized scores, because many of the DCD participants' standardized scores reached the maximum standardized score and therefore there would be little variance in the range of scores. In the DCD group, correlations with overall tactile localization accuracy were non-significant (Conners $(n=18)$ : $r$ $=-.195, p=.437$; Peg placing $(\mathrm{n}=19): \mathrm{r}=-.377, p=.112)$. This was also the case for the CAIQ group (Conners $(n=14): r=-.273, p=.345$; Peg placing $(n=19): r=.195, p=.424)$. For 
the motor matched group, the correlation for the parents who completed the ADHD Index (for age 6 years +$)$ version of the Conners was significant $(n=6)(\mathrm{r}=.857, p=.029)$.

Given the potential of the ceiling effects outlined above to produce null correlations, we conducted correlations between the individual difference measures of interest and the tactile localisation accuracy scores on the condition in which all groups' scores were significantly different from 100\%: the Unseen Crossed condition. Spearman's correlations were: DCD group (Conners, $\mathrm{n}=18: \mathrm{r}=-.570, p=.013$; peg placing, $\mathrm{n}=19: \mathrm{r}=-.295, p=$ .219); CAIQ group (Conners, $\mathrm{n}=14: \mathrm{r}=-.139, p=.636$; peg placing, $\mathrm{n}=19: \mathrm{r}=.196, p=$ $.420)$; MM group (Conners, $\mathrm{n}=6: \mathrm{r}=.490, p=.324)$.

\section{Discussion}

The primary aim of this study was to determine whether there is a more broad impairment in multisensory body representations in DCD beyond the domain of skilled sensorimotor behaviour. To do this, we examined perceptual localization of touch, a function which is known to be strongly underlain by multisensory processes, using a paradigm which would elicit the crossed hands effect in typical development (e.g., Heed \& Azanon, 2014; Rigato et al., 2014; Roder et al., 2004). Participants across all of the groups tested in this study demonstrated poorer localization of touch in the crossed hands posture. We can infer from this result that visual experience in early life has given rise to external coding of touch in DCD, as it does in typically developing individuals (see Röder et al., 2004; Ley et al., 2013). A comparison of the effect of posture on tactile localization across the groups tested here indicated no significant differences. This indicates that the development of the use of an external spatial coding of touch is robust to the developmental impairments that occur in DCD. Therefore, individuals with DCD can be expected to experience the same overall pattern of advantage for sensory processing occurring in canonical body positions, as TD individuals do. 
A second aim of our investigation was to establish whether DCD children's tactile localization performance per se (i.e., irrespective of questions about external coding of touch) is in keeping with their development, focussing particularly on whether they were in line with the performance of typically developing children matched on chronological age and cognitive development, and also a comparison of their performance with younger, motor matched children. In line with our expectations, group differences in tactile localisation ability emerged. Even though there is a negligible sensorimotor requirement in the tactile localization task presented in our study, the DCD group's performance was in line with that of younger, motor matched children rather than with their cognitive ability (and chronological age) matched peers who out-performed them across conditions. This prompts the conclusion that DCD, rather than being a specific impairment of sensorimotor functions, may reflect a somewhat broader impairment in perceptual as well as sensorimotor body representations (see Hill et al., 2012).

In Begum Ali and colleagues' (2014) study, which used the same task as the study reported here, the youngest children tested (4-year-olds) made more errors of tactile localization when their hands were visible than when their hands were hidden from sight. Begum Ali et al. argued that this finding represents a difficulty in early development with integrating visual cues concerning the limbs into representation of the layout of the limbs which informs tactile localization. This effect was not found in any of the groups tested in our study. It is unsurprising that the typically developing children did not show this effect given that our youngest group of participants were 5-6 years of age (at which point Begum Ali et al. observed no effect of limb visibility). However, the absence of this pattern of visual interference in the DCD group in the current study suggests that even though tactile localization ability in DCD appears immature, the participants with DCD were not exhibiting the characteristics of the very youngest children who have been tested with this task. Future 
work should map a complete developmental trajectory of this ability and examine where the participants with DCD fit along it, if at all (cf. Thomas et al., 2009). In addition, measuring tactile localization for adults with DCD would provide an indication of whether or not tactile localization ability 'catches up' with chronological-age peers by the time individuals reach adulthood.

One possible interpretation of the poorer tactile localization in children with DCD is that their tactile localization abilities have been limited by motor coordination difficulties in development. If this were the case, we would expect to see a direct relationship between individual children's motor skill and their tactile localization task performance. However, the lack of significant association between individual children's fine motor skill, as measured by the peg-moving task, and tactile localization ability in the DCD group suggests that the notion of tactile localization being linked purely with motor skills is too simplistic (although, the generally high accuracy on the tactile localization task may have constrained the potential for detecting a relationship here). Nonetheless, further work will be needed to determine the extent to which motor skills and tactile perceptual abilities are linked in development. For instance, it remains to be seen whether impairments in tactile localization will arise in every disorder in which there are difficulties with motor skills (motor impairments are well represented across a wide range of developmental disorders; Hill et al., 2012). There are also ongoing questions about subtypes of DCD in the current literature (Visser, 2003) which is particularly relevant here because individuals' perceptual abilities are one of the dimensions along which hypothesised subtypes of DCD rest (see Green, Chambers \& Sugden, 2008). One future direction for this line of research should be to investigate individual differences in the manifestation or otherwise of tactile localization difficulties in DCD, using a larger sample than in the current study. 
An association between Conners score and overall tactile localisation ability was present in the younger motor matched group (albeit for a small sample size), but unexpectedly, with higher (i.e., poorer) attention ratings being associated with better tactile localisation. This could be attributed to the small sample size in this analysis $(n=6)$. This association disappeared when the most challenging task condition was analysed, prompting the possibility that it is a chance finding.

The lack of significant correlation between parent-rated attention scores and overall tactile localization ability in DCD also suggests that poor performance on this task was not simply a matter of attention difficulties, a characteristic commonly reported to be associated with the disorder (e.g., Dewey, Kaplan, Crawford, \& Wilson, 2002). However, the role of attention difficulties experienced by many children with DCD has also been illustrated by the significant correlation between Conners ratings and scores on the most difficult task condition, which were not apparent for either of the typically developing groups. Although parents of children with DCD endorsed more of the items on the Conners questionnaire than parents of typically developing children, only one of the DCD sample had also received a diagnosis of attention deficit disorder. Therefore, poor scores on this tactile localisation task seem likely to arise from a combination of impaired multisensory representations and attention difficulties in our DCD sample.

In our view, the most likely explanation for poor tactile localization in DCD is a general deficit in multisensory body representations, which renders it difficult for these children to construct representations about their everyday environments in a way that is as stable and predictable as it is for a typically developing individual. One theoretical position which might inform this perspective is that of automatization deficits in DCD, and specific learning disorders more broadly (Nicolson, Fawcett, \& Dean, 2001). The 'automatization deficit hypothesis' (Fawcett \& Nicholson, 1992; Fawcett, 2002) which has been developed in 
relation to dyslexia, postulates that impairments in the cerebellum are responsible for reduced automaticity and fluency in motor skills in developmental disorders (see also Nicolson \& Fawcett, 2011). Evidence for the automatization deficit hypothesis of dyslexia comes from impairments in dual-task processing, such as balancing on a beam whilst counting (Fawcett \& Nicholson, 1992): counting disrupted balance for individuals with dyslexia but not for controls, leading to the hypothesis that the balance was less fluent for the dyslexic individuals and thus required more conscious attention, being disrupted when that attention was directed towards counting. The cerebellum is implicated not only in movement skills (e.g., Nowak et al., 2007) but also in wider multisensory processes (e.g., Christensen et al., 2014) and, therefore, could play a role in the generation of weaker multisensory representations in DCD. More specifically, if the integration of information from multiple sensory channels in DCD is less automatic than in typical development, perhaps it would require the individual to pay closer conscious attention to the process of locating a touch, finding one's balance, judging a distance, and so on. This would leave fewer resources available for processing inputs from other sensory sources, leading to degraded perceptual representations. Furthermore, the relationship between reported attentional capabilities and task performance on the most demanding condition (Unseen, Crossed) in the DCD group could be reflecting a reduced level of automatic processing of tactile localisation in the DCD group, when arms are in a noncanonical posture (crossed): if children with DCD do indeed need to pay closer conscious attention in this condition than their typically developing counterparts due to reduced automaticity, attention could serve as a limiting factor for the DCD and not TD groups, which is the correlational pattern observed. Future research would be required to investigate these hypotheses.

Some limitations of the current study constrain the weight of the conclusions that can be drawn. In terms of participant selection, it was not possible to collect measures 
determining inclusion criteria for every participant in an identical way: for example in the DCD group, Movement-ABC Checklist questionnaire data was unavailable for one participant, but we were able to use their MABC Test score to confirm motor difficulties. It was only possible for our motor matched group to be matched as a group and not on an individual basis. As peg placing is a reaction-time variable for an experimental task rather than a standardised measure of performance, we must expect some variability within groups and within participants. Thus, taking a group mean allows a comparison of peg placing performance for the group as a whole, which is likely to be robust to individual differences within the group.

Ceiling performance was observed in some of the experimental conditions in some of the groups tested. In particular, it seems likely that the CAIQ group would have been able to exhibit even greater tactile localisation ability on a task which was more demanding. However, the task paradigm was selected in order to be accessible enough for the younger TD participants and the children with DCD, and thus yield a comparison to of DCD children's performance with their TD counterparts. Ceiling effects may have limited the power of correlational analyses between some measures, which we addressed by also conducting correlations between tactile localisation accuracy and the individual differences measures of interest for the task condition for which performance was significantly different from $100 \%$ in every group. For future work it may be possible to modify the task so that reliable reaction time data could be collected, which, alongside accuracy, may provide a more nuanced picture of performance unhindered by ceiling effects.

Given our focus on multisensory processes in this report, it seems appropriate to consider the relevance of sensory integration therapy (SIT; e.g., Ayres, 1979). SIT's use is based on the assumption that children with many neurodevelopmental disorders, (not just DCD), have difficulties modulating sensory inputs. However, SIT has had limited success in 
terms of interventions (e.g., Kaplan, Polatajko, Wilson, \& Faris, 1993). The current study was not designed with the aim of informing the use of SIT, and so does not support its use per se, in light of the lack of robust evidence for its effectiveness. It may be that children with various neurodevelopmental disorders experience a range of different problems with sensory integration, and that this leads SIT to be limited in its effectiveness (Hill et al., 2012). It remains to be seen whether more targeted forms of SIT which are based on disorder-specific evidence enjoy any further success. However, other forms of intervention have been shown to be more fruitful in DCD. In a recent meta-analysis, task-oriented interventions, in which children are taught a specific skill relevant to everyday life, were shown to be more successful than process-oriented ones (of which SIT is one example), where the focus is on remediation of an underlying process which may be problematic (Smits-Engelsman et al., 2013). The relative success of task-based intervention approaches in those with DCD (for example, Cognitive Orientation to Daily Occupational Performance; CO-OP, Polatajko, Mandich, Miller, \& Macnab, 2001) may be linked to the immature multisensory integration which has been identified in this study: tasks require an individual to use many skills in concert whereas processes rely on one skill/low level ability at a time. Therefore, perhaps task-based interventions are more successful because they assist with the (late) development of multisensory integration, while process-based interventions focus on teaching the process, but not how to integrate that process with other processes. Future work could investigate these possibilities with a focus on the clinical and daily life applications of multisensory impairments in DCD.

\section{Conclusions}

In this study we have demonstrated for the first time that children with DCD, like typical controls, exhibit a crossed hands effect and therefore use an external reference frame for locating touches to the hands. Difficulties in a group of children with DCD, have also 
been revealed on tactile localization per se. Tactile localization requires integration of information from multiple sensory modalities including touch, vision, proprioception, and even hearing (e.g., Pavani, Spence, \& Driver, 2000; Rigato et al., 2013; Tajadura-Jiménez, Väljamäe, Toshima, Kimura, Tsakiris \& Kitagawa, 2012), and on this basis we suggest that difficulties in motor ability and tactile localization in children with DCD may reflect a broader impairment of multisensory body representations. Indeed there are indications that tactile localization is also impaired in other disorders where motor skill is compromised (e.g., Yoshioka, Dillon, Beck, Rapp, \& Landau, 2013). While our study provides an initial window into the tactile localization abilities of children with DCD, only future work which takes a longitudinal approach can start to address how tactile localization and sensorimotor impairments are causally related in development (Karmiloff-Smith, 1998). 


\section{ACKNOWLEDGEMENTS}

We are extremely grateful to the children with DCD and their families who took part in the study as well as children, parents and staff at Edmund Waller, Kender and Ivydale primary schools and two anonymous reviewers for their helpful comments. We are also grateful to the University of Leeds for granting us the use of research space. Thanks go to Emma Sumner and the students who provided assistance with data collection. This research is funded by European Research Council under the European Community's Seventh Framework Programme (FP7/2007-2013) / ERC Grant agreement no. 241242. The funders had no role in the study design, collection, analysis or interpretation of data. 


\section{REFERENCES}

American Psychiatric Association. (2013). Diagnostic and statistical manual of mental disorders (5th ed.)

Ayres, A. J. (1979). Sensory integration and the child. Los Angeles: Western Psychological Services.

Azañón, E., Stenner, M. P., Cardini, F., \& Haggard, P. (2015). Dynamic tuning of tactile localization to body posture. Current Biology, 25(4), 512-517.

Barnett, A., Henderson, S. E., \& Sugden, D. A. (2007). Movement Assessment Battery for Children - Second Edition (Movement ABC-2). London: Pearson.

Begum Ali, J., Cowie, D., \& Bremner, A. J. (2014). Effects of posture on tactile localization by 4 years of age are modulated by sight of the hands: evidence for an early acquired external spatial frame of reference for touch. Developmental Science, 17(6), 935-943.

Begum Ali, J., Spence, C., \& Bremner, A.J. (2015). Human infants' ability to perceive touch in external space develops postnatally. Current Biology, 25, R978-R979.

Bremner, A. J., \& de Fockert, J. (2016). Sensory Development: Childhood Changes in Visual Cortical Function. Current Biology, 26(1), R36-R37.

Bremner, A. J., Hill, E., Pratt, M., Rigato, S., \& Spence, C. (2013). Bodily illusions in young children: developmental change in the contribution of vision to perceived hand position in early childhood. PLoS ONE, 8, e51887.

Bremner, A. J., Holmes, N. P., \& Spence, C. (2008). Infants lost in (peripersonal) space? Trends in Cognitive Sciences 12(8):298-305. doi: 10.1016/j.tics.2008.05.003. Epub 2008 Jul 5. 
Bremner, A. J., Holmes, N. P., \& Spence, C. (2012). The development of multisensory representations of the body and of the space around the body. In A. Bremner, D. J. Lewkowicz \& C. Spence (Eds.), Multisensory Development. Oxford: Oxford University Press.

Bremner, A. J., Lewkowicz, D. J., \& Spence, C. (2012). The multisensory approach to development. In A. J. Bremner, D. J. Lewkowicz \& C. Spence (Eds.), Multisensory Development (pp. 1-26). Oxford: Oxford University Press.

Bremner, A. J., Mareschal, D., Lloyd-Fox, S., \& Spence, C. (2008). Spatial localization of touch in the first year of life: early influence of a visual spatial code and the development of remapping across changes in limb position. Journal of Experimental Psychology: General, 137(1), 149-162.

Bremner, A. J., \& Spence, C. (in preparation). The development of tactile perception. In J. Benson (Ed.), Advances in Child Development and Behavior, Vol. 52. Oxford, UK: Elsevier.

Bremner, A. J., \& van Velzen, J. (2015). Sensorimotor Control: Retuning the Body-World Interface. Current Biology, 25(4), 159-161.

Christensen, A., Giese, M. A., Sultan, F., Mueller, O. M., Goericke, S. L., Ilg, W., \& Timmann, D. (2014). An intact action-perception coupling depends on the integrity of the cerebellum. The Journal of Neuroscience, 34(19), 6707-6716.

Conners, K. (2008). Conners 3 ADHD Index - Parent version. US: Multi-Health Systems Inc.

Conners, K. (2009). Conners 3 Early Childhood Global Index-Parent. US: Multi-Health Systems Inc. 
Cowie, D., Makin, T., \& Bremner, A.J. (2013). Children's responses to the Rubber Hand Illusion reveal dissociable pathways in body representations. Psychological Science, 24, 762-769.

Cowie, D., Sterling, S., \& Bremner, A.J. (2016). The development of multisensory body representation and awareness continues to ten years of age: evidence from the rubber hand illusion. Journal of Experimental Child Psychology, 142, 230-238.

Cascio, C. J. (2010). Somatosensory processing in neurodevelopmental disorders. Journal of neurodevelopmental disorders, 2(2), 62-69.

Deconinck, F., De Clercq, D., Savelsbergh, G. J., Van Coster, R., Oostra, A., Dewitte, G., \& Lenoir, M. (2006). Visual contribution to walking in children with developmental coordination disorder. Child: Care, Health and Development, 32(6), 711-722.

Dekker, T. M., Ban, H., van der Velde, B., Sereno, M. I., Welchman, A. E., \& Nardini, M. (2015). Late development of cue integration is linked to sensory fusion in cortex. Current Biology, 25(21), 2856-2861.

Dewey, D., Kaplan, B. J., Crawford, S. G., \& Wilson, B. N. (2002). Developmental coordination disorder: associated problems in attention, learning, and psychosocial adjustment. Human Movement Science, 21(5), 905-918.

Dunn, L. M., Dunn, D. M., Sewell, J., Styles, B., Brzyska, B., Shamsan, Y., \& Burge, B. (2009). The British Picture Vocabulary Scale - 3rd edition (Third ed.). London: GL Assessment Limited.

Ernst, M. O., \& Banks, M. S. (2002). Humans integrate visual and haptic information in a statistically optimal fashion. Nature, 415(6870), 429-433.

Gori, M., Del Viva, M., Sandini, G., \& Burr, D. C. (2008). Young children do not integrate visual and haptic form information. Current Biology, 18(9), 694-698. 
Heed, T., \& Azañón, E. (2014). Using time to investigate space: a review of tactile temporal order judgments as a window onto spatial processing in touch. Frontiers in Psychology, 5, 76.

Hill, E. L., Crane, L., \& Bremner, A. J. (2012). Developmental disorders and multisensory perception. In A. J. Bremner, D. J. Lewkowicz \& C. Spence (Eds.), Multisensory Development (pp. 273-300). Oxford: Oxford University Press.

Karmiloff-Smith, A. (1998). Development itself is the key to understanding developmental disorders. Trends in Cognitive Sciences, 2(10), 389-398.

Laszlo, J. I., Bairstow, P. J., Bartrip, J., \& Rolfe, U. T. (1988). Clumsiness or PerceptuoMotor Dysfunction? Advances in Psychology, 55, 293-310.

Lord, R., \& Hulme, C. (1987). Kinaesthetic Sensitivity Of Normal And Clumsy Children. Developmental Medicine \& Child Neurology, 29(6), 720-725. doi: 10.1111/j.14698749.1987.tb08816.x

Medina, J., \& Coslett, H. B. (2010). From maps to form to space: touch and the body schema. Neuropsychologia, 48(3), 645-654.

Mon-Williams, M. A., Wann, J. P., \& Pascal, E. (1999). Visual-proprioceptive mapping in children with developmental coordination disorder. Developmental Medicine \& Child Neurology, 41(4), 247-254.

Nardini, M., Bedford, R., \& Mareschal, D. (2010). Fusion of visual cues is not mandatory in children. Proceedings of the National Academy of Sciences, 107(39), 17041-17046.

Nardini, M., Begus, K., \& Mareschal, D. (2013). Multisensory uncertainty reduction for hand localization in children and adults. Journal of Experimental Psychology: Human Perception and Performance, 39(3), 773.

Nardini, M., Jones, P., Bedford, R., \& Braddick, O. (2008). Development of cue integration in human navigation. Current Biology, 18(9), 689-693. 
Nicolson, R. I., Fawcett, A. J., \& Dean, P. (2001). Developmental dyslexia: the cerebellar deficit hypothesis. Trends in Neurosciences, 24(9), 508-511.

Nicolson, R. I., \& Fawcett, A. J. (2011). Dyslexia, dysgraphia, procedural learning and the cerebellum. Cortex 47, 117-127.

Nowak, D. A., Topka, H., Timmann, D., Boecker, H., \& Hermsdörfer, J. (2007). The role of the cerebellum for predictive control of grasping. The Cerebellum, 6(1), 7-17.

Pagel, B., Heed, T., \& Röder, B. (2009). Change of reference frame for tactile localization during child development. Developmental Science, 12(6), 929-937. doi: 910.1111/j.1467-7687.2009.00845.x.

Raven, J. (2004). Raven's Coloured Progressive Matrices. London: Pearson.

Röder, B., Rosler, F., \& Spence, C. (2004). Early vision impairs tactile perception in the blind. Current Biology, 14(2), 121-4.

Schicke, T., \& Röder, B. (2006). Spatial remapping of touch: confusion of perceived stimulus order across hand and foot. Proceedings of the National Academy of Sciences, 103(31), 11808-11813.

Schoemaker, M. M., van der Wees, M., Flapper, B., Verheij-Jansen, N., Scholten-Jaegers, S., \& Geuze, R. H. (2001). Perceptual skills of children with developmental coordination disorder. Human Movement Science, 20 (1), 111-133.

Shore, D. I., Spry, E., \& Spence, C. (2002). Confusing the mind by crossing the hands. Cognitive Brain Research 14(1), 153-63.

Sinani, C., Sugden, D. A., \& Hill, E. L. (2011). Gesture production in school vs. clinical samples of children with Developmental Coordination Disorder (DCD) and typically developing children. Research in developmental disabilities, 32(4), 1270-1282.

Smits-Engelsman, B. C. M., Blank, R., Van der Kaay, A., Mosterd van der Meijs, R., Vlugt van den Brand, E., Polatajko, H. J., \& Wilson, P. H. (2013). Efficacy of interventions 
to improve motor performance in children with developmental coordination disorder: a combined systematic review and meta- analysis. Developmental Medicine \& Child Neurology, 55(3), 229-237.

Sparto, P. J., Redfern, M. S., Jasko, J. G., Casselbrant, M. L., Mandel, E. M., \& Furman, J. M. (2006). The influence of dynamic visual cues for postural control in children aged 7-12 years. Experimental Brain Research, 168(4), 505-516.

Spence, C., \& Driver, J. (2004). Crossmodal space and crossmodal attention: Oxford University Press.

Sugden, D. A., \& Henderson, S. E. (2007). Movement Assessment Battery for Children Checklist - Second Edition (Movement ABC-2 Checklist). London: Pearson.

Tajadura-Jiménez, A., Väljamäe, A., Toshima, I., Kimura, T., Tsakiris, M., \& Kitagawa, N. (2012). Action sounds recalibrate perceived tactile distance. Current Biology, 22(13), R516-R517.

Thomas, M. S. C., Annaz, D., Ansari, D., Scerif, G., Jarrold, C., \& Karmiloff-Smith, A. (2009). Using developmental trajectories to understand developmental disorders. Journal of Speech, Language, and Hearing Research, 52(2), 336.

Visser, J. (2003). Developmental coordination disorder: a review of research on subtypes and comorbidities. Human Movement Science, 22(4-5), 479-493. doi: 10.1016/j.humov.2003.09.005

von Hofsten, C., \& Rösblad, B. (1988). The integration of sensory information in the development of precise manual pointing. Neuropsychologia, 26(6), 805-821.

Wann, J. P., Mon-Williams, M., \& Rushton, K. (1998). Postural control and co-ordination disorders: The swinging room revisited. Human Movement Science, 17(4-5), 491513. doi: http://dx.doi.org/10.1016/S0167-9457(98)00011-6 
Yamamoto, S., \& Kitazawa, S. (2001). Reversal of subjective temporal order due to arm crossing. Nature neuroscience, 4(7), 759-765.

Yoshioka, T., Dillon, M. R., Beck, G. C., Rapp, B., \& Landau, B. (2013). Tactile Localization on Digits and Hand Structure and Development. Psychological science, 24(9), 1653-1663. 


\section{Figures}
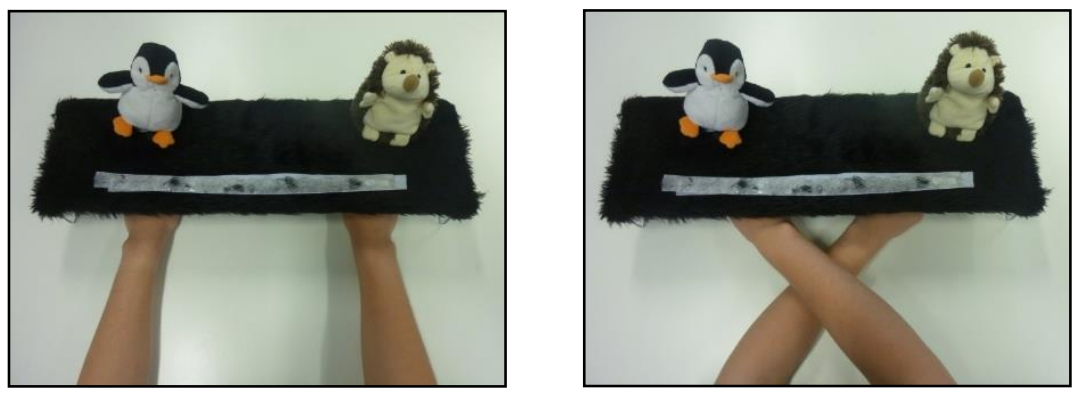

Figure 1: example of seen, uncrossed condition set-up (left hand side) and seen, crossed condition set-up (right hand side) from the participant's point of view. In the unseen conditions, a black cloth of the same faux fur fabric covered the participants' arms. 


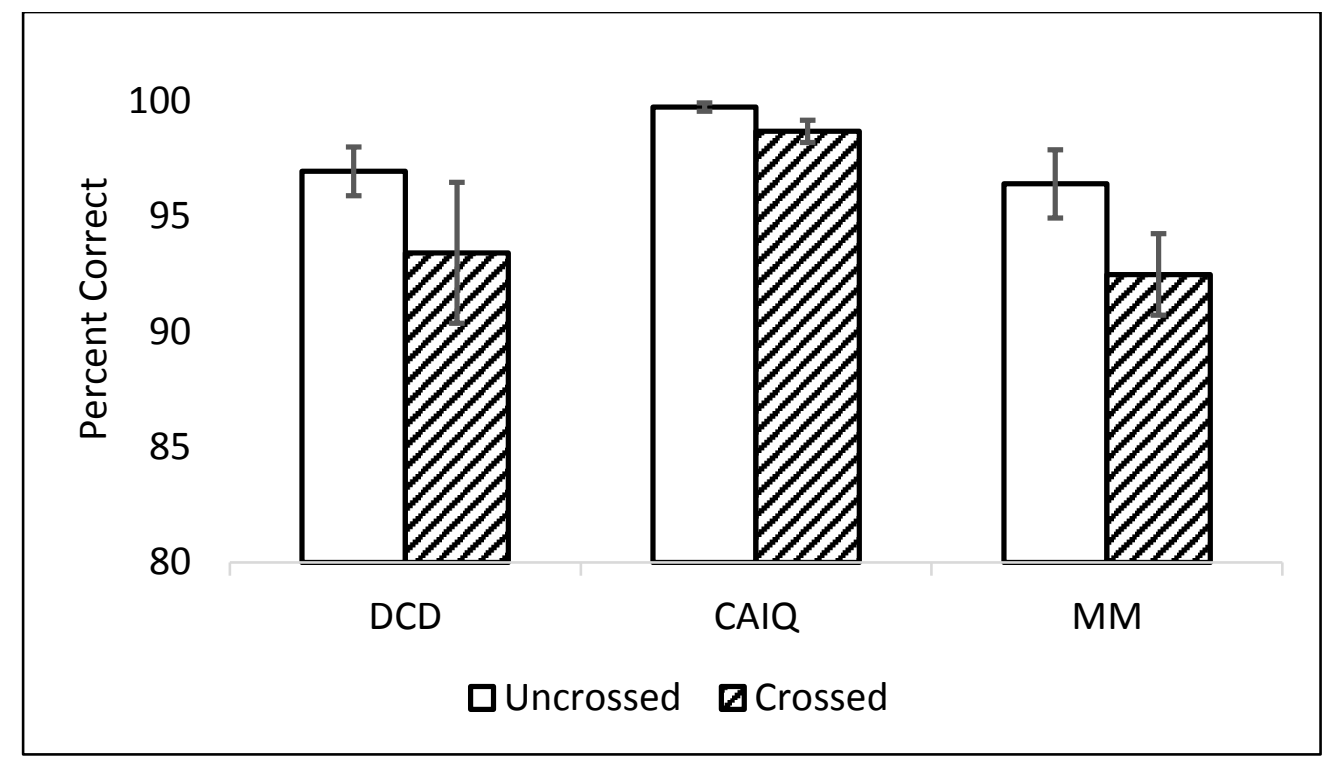

Figure 2: Group means of percentage accuracy for DCD, CAIQ and Motor matched groups, for the mean of the Crossed conditions and the mean of the Uncrossed conditions. Error bars denote the standard error of the mean. DCD = developmental coordination disorder group, CAIQ = group matched on chronological age and cognitive ability, $\mathrm{MM}=$ motor matched group. 


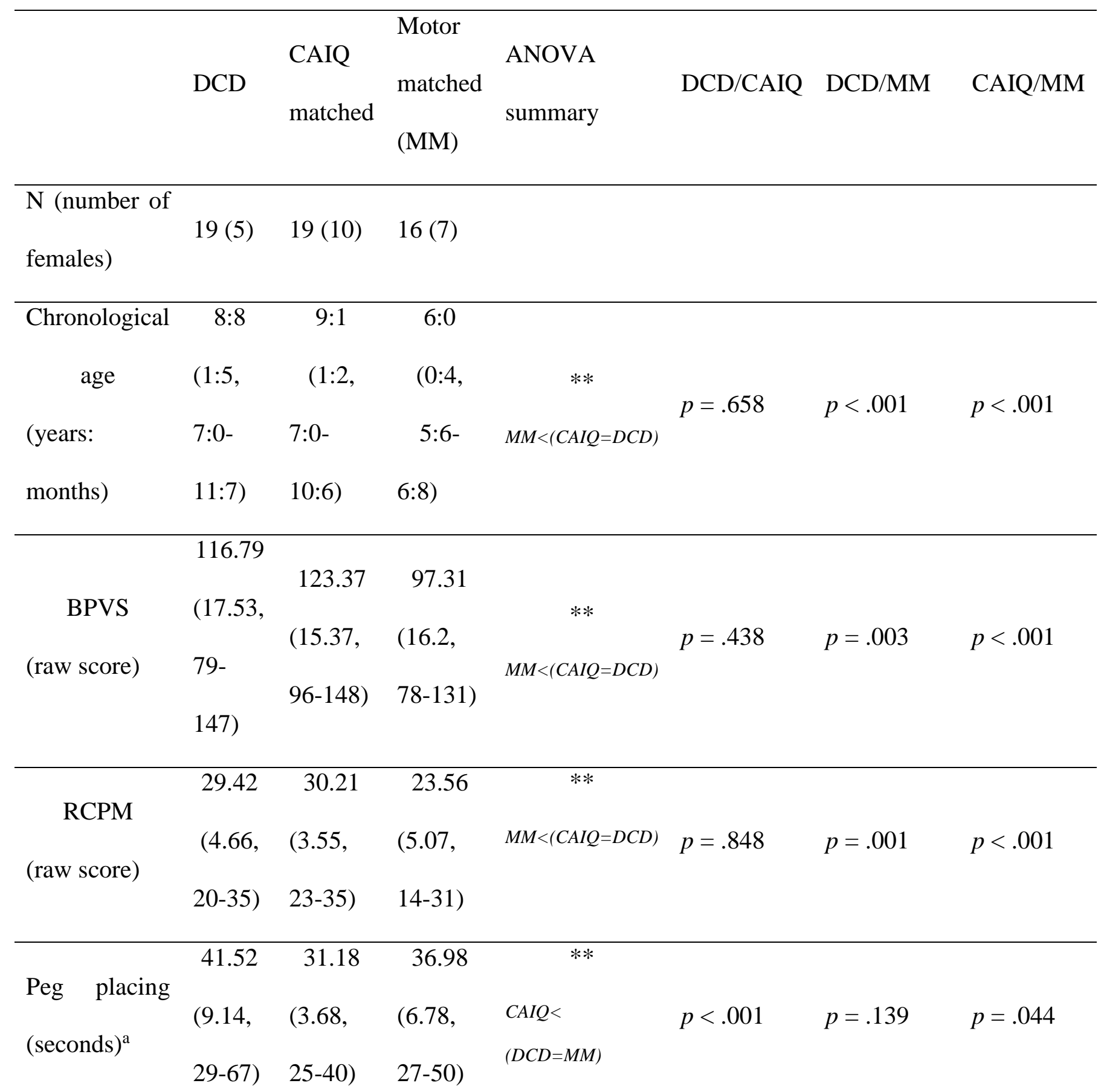

Note. BPVS = British Picture Vocabulary Scale. RCPM = Raven's Coloured Progressive Matrices. DCD = developmental coordination disorder group. $\mathrm{CAIQ}=$ group matched on chronological age and cognitive ability. $\mathrm{MM}=$ motor matched group. $* *=$ one-way ANOVA significant at $p<.001$. DCD/CAIQ, DCD/MM and CAIQ/MM columns contain relevant Tukey post hoc $p$ values for group comparisons. ${ }^{a}$ Peg placing minimums and maximums are rounded to the nearest whole second.

Table 1: Means (standard deviations, ranges) of participant demographics. $\mathrm{CA}: \mathrm{F}(2,51)=$ 39.927, $\mathrm{y}_{\mathrm{p}}^{2}=0.61 ; \operatorname{BPVS}: \mathrm{F}(2,51)=11.613, \mathrm{y}_{\mathrm{p}}^{2}=.313 ; \operatorname{RCPM}: \mathrm{F}(2,51)=11.336, \mathrm{y}_{\mathrm{p}}^{2}=.308$;

Peg placing: $\mathrm{F}(2,51)=10.677, \mathrm{y}^{2} \mathrm{p}=.295$. 


\begin{tabular}{|c|c|c|c|c|c|c|}
\hline & \multirow{2}{*}{$\mathrm{n}$} & Seen, & Seen, & Unseen, & Unseen, & Overall \\
\hline & & Uncrossed & Crossed & Uncrossed & Crossed & accuracy \\
\hline \multirow[b]{2}{*}{ DCD } & \multirow[b]{2}{*}{19} & 97.59 & 94.74 & 96.32 & 92.11 & 95.19 \\
\hline & & (3.9) & $(9.93)$ & (7.61) & (18.13) & (7.2) \\
\hline \multirow{2}{*}{ CAIQ } & \multirow{2}{*}{19} & 100.00 & 98.68 & 99.47 & 98.68 & 99.21 \\
\hline & & $(0.00)$ & $(3.27)$ & (1.58) & $(2.26)$ & (1.33) \\
\hline \multirow[b]{2}{*}{ MM } & \multirow[b]{2}{*}{16} & 95.00 & 93.40 & 97.81 & 91.56 & 94.44 \\
\hline & & (10.33) & $(7.02)$ & $(4.46)$ & $(9.26)$ & $(4.80)$ \\
\hline
\end{tabular}

Note. DCD = developmental coordination disorder group, CAIQ = group matched on chronological age and cognitive ability, MM = motor matched group.

Table 2: Means (standard deviation) of percentage accuracy by condition and group. 